\title{
Fast and reliable control of steering mirrors with application to free-space communication
}

\author{
Salim Ibrir \\ Electrical Engineering Department \\ King Fahd University \\ of Petroleum and Minerals \\ Dhahran, 31261, KSA \\ Email: sibrir@kfupm.edu.sa
}

\author{
Chun-Yi Su \\ College of Mechanical Engineering \\ and Automation \\ Huaqiao University, \\ Xiamen 361021, China
}

\author{
Boon S. Ooi and M. Slim Alouini \\ Computer, Electrical and Mathematical \\ Science and Engineering Division \\ King Abdullah University \\ of Science and Technology, KSA \\ Email: \{boon.ooi, slim.alouini\}@kaust.edu.sa
}

\begin{abstract}
After modeling a laser beam pointing system actuated by a piezo-electric fast steering mirror (FSM), an observerbased linear feedback controller is developed to correct the position of the laser spot communicated by the position sensing detector (PSD). The modeling and the control actions have been considered with and without the hysteresis effect induced by the piezoelectric actuators of the FSM. The design of the feedback is given through the solution of parametric matrix inequalities. It was found that the integral feedback design is very efficient in handling input and system uncertainty. The linearity of the observer-based feedback has facilitated both the real-time implementation of the control strategy and the proof of stability using dynamic-output feedbacks.
\end{abstract}

Keywords-Laser pointing and control; Fast Steering Mirrors; Mechatronics; Robust Optimization.

\section{INTRODUCTION}

The Free-Space-Optical (FSO) communication is a modern and a promising optical-based communication technology. This comes from the fact that FSO communication principle is not basically dependent on the physical connections and installation to set a line of communication between the transmitter and the receiver. More precisely, FSO technology uses the free space as the air or the outer space as a media for propagation of light containing the transmission data. The absence of the signal guide in this kind of technology may facilitate the communication between objects that may move from place to place or objects that are far from each other. The nice properties of FSO transmission and reception make this mode of communication very efficient and useful when the physical connections are impractical due to design impossibilities, far distance, high costs or other limitations. Due to the aforementioned features, FSO communication seems, for instance, an excellent alternative to classical technologies that usually employ optical fiber cables or optical transmission lines. However, the primary limitations of laser communication are generally caused by the weather effects and the pointing requirements. In order to maintain an excellent and reliable communication, the transmitting source and the receiver module should ensure and maintain an accurate pointing and tracking of the laser communication beam. To overcome this inherent difficulties, intelligent control systems have been proposed to track and filter the beams for reliable communication.

In FSO communication, the laser is used to carry the low frequency signals as schematized in Fig. 1. In addition, the transmitter and the receiver may be in different situations depending on the constraints of the applications, see figures 2-5. Even when the transmitter and the receiver are fixed, the laser beam that contains the modulated signals is in need for a precise alignment and correction to ensure a good demodulation of the transmitted signal at the receiver side. Referring to the existing literature, a great deal of interest has been devoted to the control of fast steering mirrors that have been used as key tools for laser beam alignment and correction, see e.g., [1], [2], [3], [4], [5]. Laser-tracking measurement systems based on a vision-guiding device has been proposed in [6]. Laser beam jitter control using recursive-least-squares adaptive filters is discussed in [7]. Other interesting contributions to laser alignment, tracking and control may be traced in [8], [9], [10], [11], [12].

In this paper, a straightforward methodology is developed for precise and reliable control of FSMs using dynamic output feedbacks. Throughout this paper, we assume that the transmitter and the receiver are fixed and they are not necessarily located on the same direction. The conditions of the experiments are set to mimic possible indoor FSO applications without any effect of temperature or air turbulence. After modeling the complex optical system as a SISO system and developing a dynamic output feedback to steer the laser spot to the origin of the PSD, the theoretical study is subsequently followed by a real-time implementation of the control strategy. It is shown that integral feedback design is very efficient for laser beam positioning under input and system uncertainties.

\section{THE EXPERIMENT}

\section{A. Motivation}

On the surface of earth, the optical communication is in most cases done via optical fibers. Optical fibers allow the transmission over relatively large distances without excessive power losses, alignment issues, and atmosphere turbulence. However, it was found that it is possible to transmit the information optically via free space or through water without exploiting any kind of waveguides to direct the signals. For large transmission distances, it is essential to direct the energy of the sender accurately in the form of a well-collimated laser beam in order to limit the often still very large loss of power between the sender and the receiver. In the meantime, the high directionality requires an accurate alignment of the transmitter 

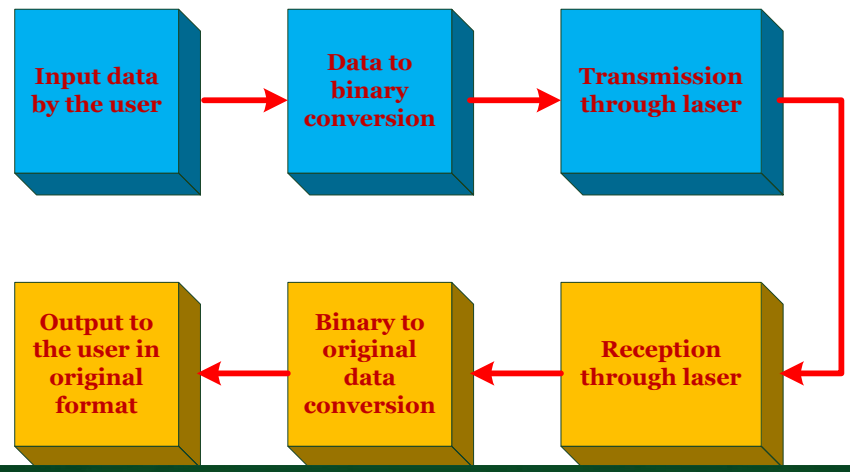

\section{MOVING-TRANSMITTER-FIXED-RECEIVER} PLATFORM
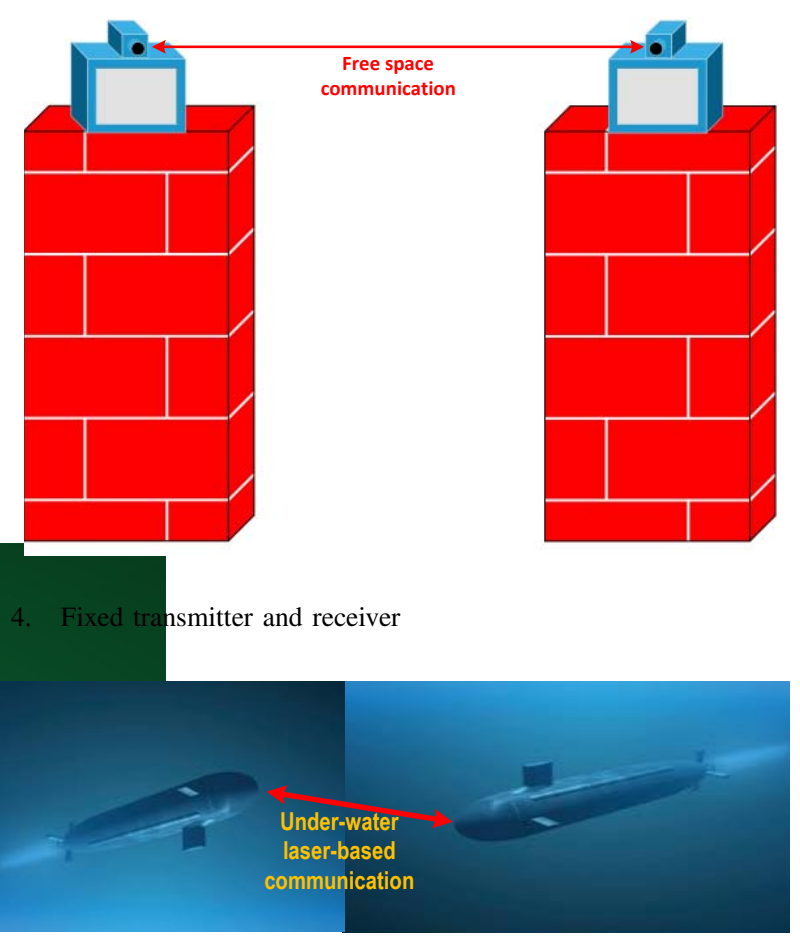

Fig. 5. Under-water free space communication

and the receiver. Therefore, it is often necessary to correct and stabilize the alignment with intelligent control systems. The use of adaptive optics to increase the directionality is one of the possible solutions that gave acceptable performances. As a matter of fact, the small dimensions of the receivers has also created a clear need to design precise and micro-positioning alignment systems to focus the laser beam on a very small active area. It this paper, a special emphasis is given to the design and the assessment of the corrective systems that allow beam positioning in a very small sensitive area under system and inpert uncertainties.

Fig. 2. Vibrations at the transmitter side

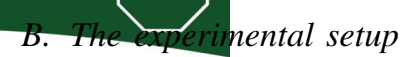

The experimental setup is composed by $0.8 \mathrm{~mW}$ SelfContained $(632.8 \mathrm{~nm}) \mathrm{HeNe}$ Laser source that integrates a red $(632.8 \mathrm{~nm}) \mathrm{HeNe}$ laser tube with a built-in voltage transformer. The rectangular housing incorporates a hard-sealed internal mirror and plasma tube design that maximizes the lifetime of the laser. The source emits a laser that hits the surface of a Polaris Kinematic Mirror with three Piezoelectric Adjusters (Thorlab POLARIS-K1PZ). The fully Integrated piezoelectric elements provide step sizes down to $0.5 \mu \mathrm{rad}$. Additionally, the matched actuator and back plate provide smooth manual kinematic adjustment. Polaris optic bores are precision machined to achieve a fit that will provide optimum beam pointing stability performance over changing environmental conditions such as temperature changes, transportation shock, and vibration. The Thorlab's piezoelectric controllers provide precise, low-noise output voltages for fine movement of piezoelectric actuators and stacks. Each output channel is independently controllable and provides a voltage ranging from $0-75 \mathrm{~V}, 0-100 \mathrm{~V}$, or $0-150 \mathrm{~V}$, depending upon the position of a switch on the back panel. The PDQ80A (PSD) detector is a segmented,

Fig. 3. Moving transmitter and fixed receiver 


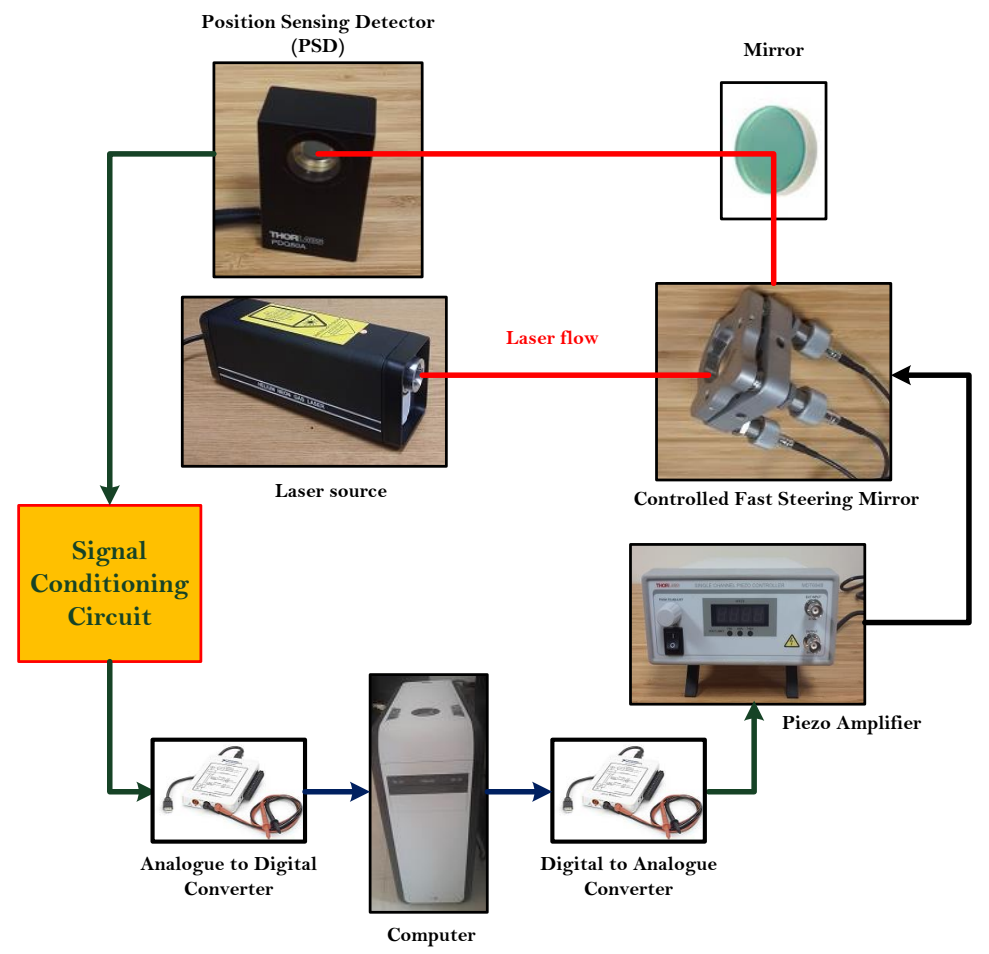

Fig. 6. Laboratory experiment

position-sensing, quadrant detector for precise path alignment of light in the 400 to $1050 \mathrm{~nm}$ or 1000 to $1700 \mathrm{~nm}$ range, respectively. A 6-pin Hirose connector on each detector outputs signals proportional to the incident beam's power distribution (and thus position).

\section{System identification}

The experimental setup is prepared as shown in Fig. 6. After fixing manually the position of the FSM to reflect the laser beam into the active area of the PSD, only one input is used to correct the position of the beam. The system to be identified is seen as the overall optical system that links the input of the FSM to the final position of the laser beam on the PSD. Since the PSD is a quadrant photo diode, the position of the beam is read in terms of two information that are the $X$ and the $Y$ positions. To set the system as a single-inputsingle-output system, the output of the system, noted $y(t)$, is selected as the square root of the squares of $X$ and $Y$. The system to be identified is selected as

$$
\mathscr{L}(y(t))=\frac{\left(\theta_{1}+\theta_{2} s+\theta_{3} s^{2}\right) \mathscr{L}(u(t))}{\theta_{4} s^{3}+\theta_{5} s^{2}+\theta_{6} s+\theta_{7}}+\theta_{8}
$$

where $\mathscr{L}(u(t))$ and $\mathscr{L}(y(t))$ are the Laplace transforms of the input and the output while $\theta_{x}=\left(\theta_{1} \cdots \theta_{7}\right)$ and $\theta_{y}=\theta_{8}$ are the constant parameters to be identified. For the zero input, we assume that the laser beam is located somewhere on the PSD quadrant. Using the peizo amplifier, the range of the applied inputs could vary from 0 to $150 \mathrm{~V}$. In this experiment, only positive voltages are tolerated by the piezoelectric actuator. To identify the system parameters, a smooth time-varying signal input is generated by Labview software and forwarded to the Thorlab amplifier through National Instrument data acquisition device myDAC. The measurement of the beam position is read by NI myDAC device and visualized in Labview. The inputoutput data are subsequently sent to the system identification toolbox of Matlab to identify all the system parameters.

\section{Controller design}

The system under consideration is modeled by the statespace equations:

$$
\begin{aligned}
& \dot{x}=A\left(\theta_{x}\right) x+B u, \\
& y=C x+\theta_{y},
\end{aligned}
$$

where $x \in \mathbb{R}^{n}(n=3)$ is the state vector, $u \in \mathbb{R}$ is one of the three possible voltage inputs applied to the mirror piezo actuator, and $y \in \mathbb{R}$ is the square root of $X^{2}+Y^{2}$ where $X$ and $Y$ are the coordinates of the beam that are measured by the PSD. The system states do not have any physical meaning since the model obtained from the identification process may have any order and may take any state-space structure. The parameters $\theta_{x}$ and $\theta_{y}$ are assumed to be constants with wellknown upper bounds. Let $z=\dot{x}$ then,

$$
\left(\begin{array}{c}
\dot{y} \\
\dot{z}
\end{array}\right)=\left(\begin{array}{cc}
\mathbf{0} & C \\
\mathbf{0} & A
\end{array}\right)\left(\begin{array}{l}
y \\
z
\end{array}\right)+\left(\begin{array}{c}
\mathbf{0} \\
B
\end{array}\right) \dot{u} \text {. }
$$

If we note $\xi=\left(\begin{array}{c}y \\ z\end{array}\right)$; this yields,

$$
\begin{aligned}
\dot{\xi} & =F\left(\theta_{x}\right) \xi+G \dot{u}, \\
y & =H \xi,
\end{aligned}
$$


where

$$
F\left(\theta_{x}\right)=\left(\begin{array}{ll}
\mathbf{0} & C \\
\mathbf{0} & A
\end{array}\right), G=\left(\begin{array}{l}
\mathbf{0} \\
B
\end{array}\right), H=\left(\begin{array}{ll}
I & \mathbf{0}
\end{array}\right) .
$$

Let

$$
\dot{\hat{\xi}}=F_{0} \hat{\xi}+G \dot{u}+L H(\hat{\xi}-\xi)
$$

be the dynamics state estimator where $F_{0}$ and $L$ are some design matrix parameters to be determined. The objective is to design an observer-based feedback of the form $\dot{u}=K \hat{\xi}$ that steers the system output $y=H \xi$ to the origin. By forcing the system output to zero, the laser beam will be focused on the center of PSD quadrants. Let's denote by $e=\hat{\xi}-\xi$ the error between the true states and the estimated ones; this immediately gives

$$
\begin{aligned}
\left(\begin{array}{c}
\dot{\xi} \\
\dot{e}
\end{array}\right) & =\left(\begin{array}{cc}
F\left(\theta_{x}\right)+G K & G K \\
F_{0}-F\left(\theta_{x}\right) & F_{0}+L H
\end{array}\right)\left(\begin{array}{l}
\xi \\
e
\end{array}\right) \\
& =A_{c l}\left(\begin{array}{c}
\xi \\
e
\end{array}\right) .
\end{aligned}
$$

It's evident that if the controller gain $K$ and the observer gain $L$ are selected such that the composite system (7) is stable then the laser beam is centered. Let $P_{1} \in \mathbb{R}^{n \times n}$ and $P_{2} \in \mathbb{R}^{n \times n}$ be two positive definite matrices and let $P=\operatorname{diag}\left(P_{1}, P_{2}\right)$. This implies that $A_{c l}$ is Hurwitz if the following matrix inequality holds true; that is: $A_{c l}^{\prime} P+P A_{c l}<0$. More explicitly, the last inequality is rewritten as follows:

$$
\left(\begin{array}{cc}
\operatorname{He}\left(P_{1} F\left(\theta_{x}\right)+P_{1} G K\right) & \left(F_{0}^{\prime}-F^{\prime}\left(\theta_{x}\right)\right) P_{2}+P_{1} G K \\
\star & \operatorname{He}\left(\left(F_{0}+L H\right)^{\prime} P_{2}\right) \\
<0 .
\end{array}\right)
$$

In order to make the last inequality numerically tractable, we shall decouple inequality (8) into two separate inequalities by introducing two arbitrary positive parameters $\varepsilon_{1}$ and $\varepsilon_{2}$ such that the following hold simultaneously:

$$
\begin{gathered}
\left(\begin{array}{cc}
\operatorname{He}\left(P_{1} F\left(\theta_{x}\right)+P_{1} G K\right)+\varepsilon_{1} I & P_{1} G K \\
\star & \operatorname{He}\left(P_{2}\left(F_{0}+L H\right)\right)+ \\
\varepsilon_{2} I &
\end{array}\right) \\
\left(\begin{array}{cc}
-\varepsilon_{1} I & \left(F_{0}^{\prime}-F^{\prime}\left(\theta_{x}\right)\right) P_{2} \\
\star & -\varepsilon_{2} I
\end{array}\right)<0 .
\end{gathered}
$$

One could verify that if (9a) and (9b) hold simultaneously, then by summing the two inequalities, inequality (8) is recovered. To reduce the number of the unknown variables, let's set $\bar{F}=P_{2} F_{0}, L=P_{2}^{-1} Y_{0}$ where $\bar{F}$ and $Y_{0}$ are the new matrix variables with appropriate dimensions. Inequalities (9a) and (9b) takes the new form:

$$
\begin{gathered}
\left(\begin{array}{cc}
\operatorname{He}\left(P_{1} F\left(\theta_{x}\right)+P_{1} G K\right)+\varepsilon_{1} I & P_{1} G K \\
\star & \left.\operatorname{He}\left(\bar{F}+Y_{0} H\right)\right)+ \\
& \varepsilon_{2} I
\end{array}\right) \\
\left(\begin{array}{cc}
-\varepsilon_{1} I & \bar{F}^{\prime}-F^{\prime}\left(\theta_{x}\right) P_{2} \\
\star & -\varepsilon_{2} I
\end{array}\right)<0
\end{gathered}
$$$$
<0 \text {, (10a) }
$$

Finally, the observer-based problem is solved numerically by solving the bilinear matrix inequalities (10a) and (10b) with respect to $P_{1}, P_{2}, K, Y_{0}$ and $\bar{F}$. The matrices of the observer are consequently computed as $L=P_{2}^{-1} Y_{0}$ and $F_{0}=P_{2}^{-1} \bar{F}$. To find a numerical solution, PENBMI computer program for solving optimization problems with quadratic objective and bilinear matrix inequality constraints is used together with MPT3. Recall that MPT3 is the Multi-Parametric Toolbox and Matlab-based toolbox for parametric optimization, computational geometry and model predictive control.

\section{HYSTERESIS EFFECT}

In this section, the hysteresis effect is taken into account while modeling the composite laser optical system. As shown in Fig. 7, the relation between the voltage applied to the piezo actuator and the deflection angle of the mirror is in form of a hysteresis. For sake of simplicity, we assume that the hysteresis output $H(u)$ is a backlash-hysteresis operator which is not explicitly measured and its parameters are not explicitly defined. However, the upper bounds of the hysteresis parameters are assumed to be well known. In order to stabilize the beam spot under this new input consideration, a suitable dynamical model is necessary to build a feedback controller. Therefore, a new formulation of the hysteresis input is needed. As a matter of fact, the hysteresis operator can be defined by:

$$
\begin{cases}\rho\left(u(t)-B_{r}\right), & \text { if; } \dot{u}>0 \\ \rho\left(u(t)-B_{l}\right), & \text { if; } \dot{u}<0 \\ & \text { and } H(u(t))=\rho\left(u(t)-B_{r}\right), \\ H\left(u\left(t_{-}\right)\right), & \text {Otherwise. }\end{cases}
$$

where, $\rho$ and $B_{r}$ are uncertain positive scalars, and $B_{l}$ is unknown negative real. The notation $H\left(u\left(t_{-}\right)\right)$means that the hysteresis output $H(u(t))$ preserves its previous value when $\dot{u}(t)=0$. As it has been reported in [13], [14], the hysteresis $H(u(t))$ can be rewritten as

$H(u(t))=\sigma_{r}(t) \rho\left(u(t)-B_{r}\right)+\sigma_{l}(t) \rho\left(u(t)-B_{l}\right)+\sigma_{s}(t) u_{s}$

where,

$$
\begin{aligned}
& \sigma_{r}(t)= \begin{cases}1, & \text { if } ; \dot{u}>0, \\
0, & \text { otherwise, }\end{cases} \\
& \sigma_{l}(t)= \begin{cases}1, & \text { if } ; \dot{u}<0, \\
0, & \text { otherwise, }\end{cases} \\
& \sigma_{s}(t)= \begin{cases}1, & \text { if } ; \dot{u}=0, \\
0, & \text { otherwise. }\end{cases}
\end{aligned}
$$

The term $u_{s}$ in (12) is a generic constant verifying the following inequality:

$$
\rho\left(u(t)-B_{r}\right) \leq u_{s} \leq \rho\left(u(t)-B_{l}\right) .
$$

Note that the above functions satisfy the equality: $\sigma_{r}(t)+$ $\sigma_{l}(t)+\sigma_{s}(t)=1$ for all $t$. According to (12) and (16), the hysteresis $H(u)$ can be written at any instant of time $t$ as follows

$$
\rho u(t)+d(u(t))
$$

where, $d(u)$ is given by:

$d(u(t))= \begin{cases}-\rho B_{r}, & \text { if; } \dot{u}>0, \\ -\rho B_{l}, & \text { if; } \dot{u}<0, \\ \xi(u(t)), & \text { if; } \dot{u}=0,-\rho B_{r} \leq \xi(u(t)) \leq-\rho B_{l} .\end{cases}$ 
a. While the POLARIS-K1PZ was physically disconnected from its piezo controller (zero bias), the ambient

temperature was increased by $15{ }^{\circ} \mathrm{C}$, then allowed to return to room temperature.

b. Measured by incrementing and decrementing the voltage applied by a TPZ001 controller in $0.1 \mathrm{~V}$ steps.

\section{Hysteresis Curves}

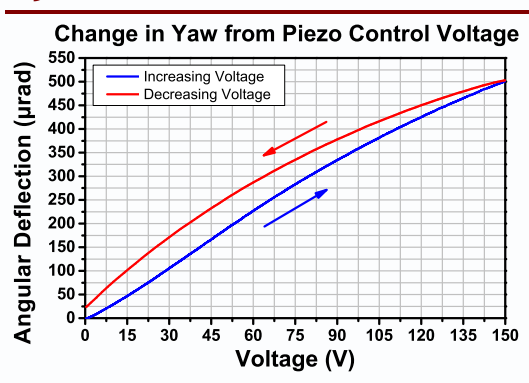

Fig. 7. Angular deflection versus voltage control input

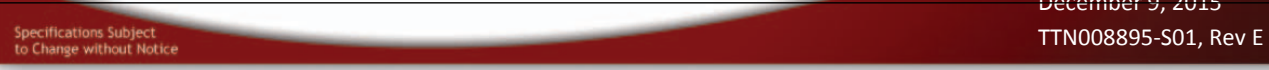

The dynamical system that relates the hysteretic input voltage $H(u)$ to the laser beam position on the PSD is now given by:

$$
\begin{aligned}
& \dot{x}=A\left(\theta_{x}\right) x+B H(u), \\
& y=C x+\theta_{y} .
\end{aligned}
$$

As a result, under the notation: $\dot{x}=z$, we have

$$
\begin{aligned}
& \dot{y}=C z, \\
& \dot{z}=A\left(\theta_{x}\right) z+B \dot{H}(u) .
\end{aligned}
$$

Actually, for $\dot{u} \neq 0$ and under the new hysteresis formulation, one could write:

$$
\begin{aligned}
& \dot{y}=C z, \\
& \dot{z}=A\left(\theta_{x}\right) z+\rho B \dot{u} .
\end{aligned}
$$

If $\dot{u}$ is seen as the new control input, it is then clear that system (21) is not controllable for $\dot{u}=0$ for which the hysteresis output $H(u)$ preserves its previous value. To overcome the uncertainty coming from the hysteresis input, a similar observer-based feedback could be conceived after adding an integrator to system (21). More explicitly, if we note $\dot{u}=v$ and $\ddot{u}=w$ with $w$ is seen now as the new control input, we have

$$
\begin{aligned}
& \dot{y}=C z, \\
& \dot{z}=A\left(\theta_{x}\right) z+\rho B v, \\
& \dot{v}=w .
\end{aligned}
$$

In matrix form, system (22) is rewritten as

$$
\begin{aligned}
\dot{\zeta} & =\left(\begin{array}{ccc}
\mathbf{0} & C & \mathbf{0} \\
\mathbf{0} & A\left(\theta_{x}\right) & \rho B \\
\mathbf{0} & \mathbf{0} & \mathbf{0}
\end{array}\right) \zeta+\left(\begin{array}{l}
\mathbf{0} \\
\mathbf{0} \\
I
\end{array}\right) w, \\
& =\boldsymbol{F}\left(\theta_{x}, \rho\right) \zeta+\boldsymbol{G} \ddot{u}, \\
y & =\left(\begin{array}{lll}
I & \mathbf{0} & \mathbf{0}
\end{array}\right) \zeta, \\
& =\boldsymbol{H} \zeta .
\end{aligned}
$$

System (23) could be stabilized in the same manner as we have proceeded with system (4). By following the same steps of the previous developed procedure, an observer-based feedback is similarly designed as $\ddot{u}=\boldsymbol{K} \hat{\zeta}$ where $\hat{\zeta}$ is the state vector of the observer:

$$
\hat{\zeta}=\boldsymbol{F}_{0} \hat{\zeta}+\boldsymbol{G} w+\boldsymbol{L} \boldsymbol{H}(\hat{\zeta}-\zeta)
$$
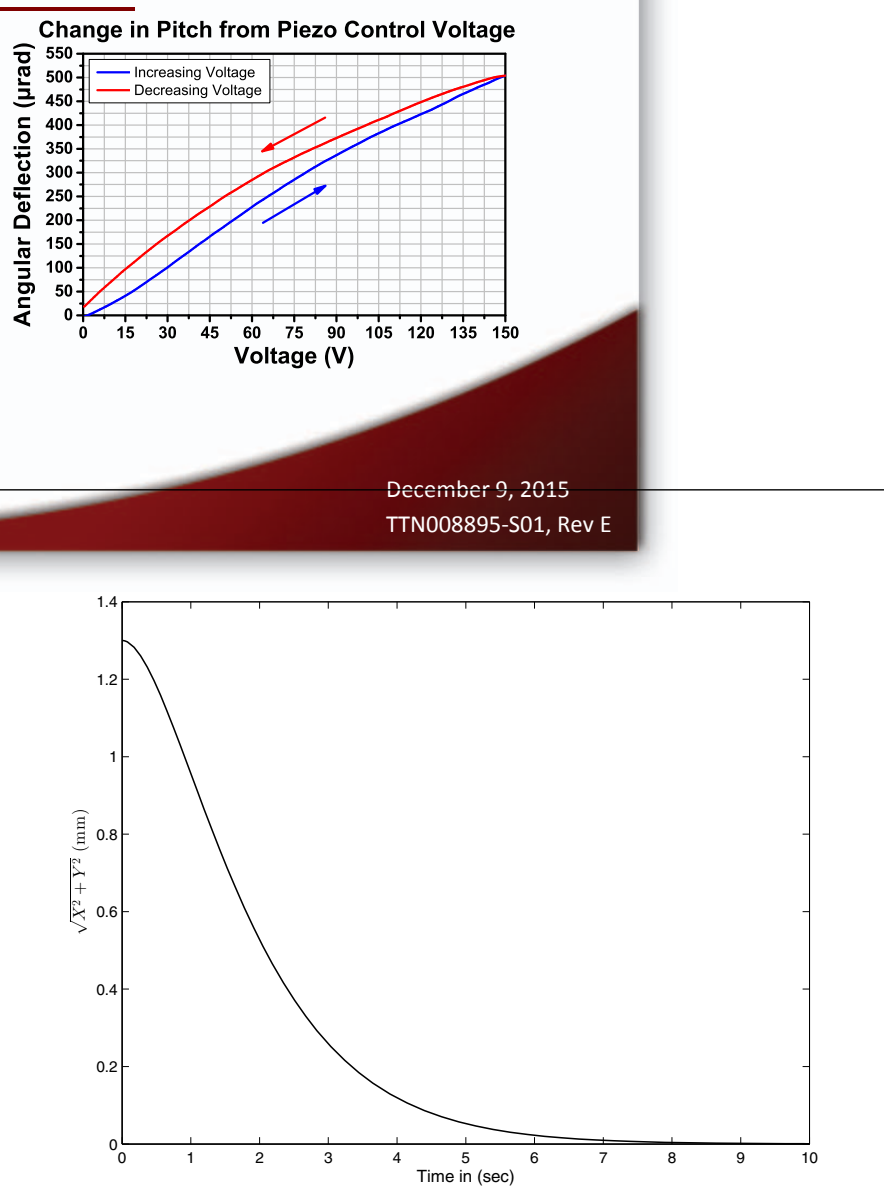

Fig. 8. The time evolution of the radius of the beam spot

\section{Simulations}

The performance of the control strategy is visualized through Figures 8,9 and 10. It is noticed that the integral action of the control feedback has eliminated error modeling and measurement errors as well. For space limitation, other simulations are not included. It is important to highlight here that the control methodology has been tested for different initial conditions as shown in Fig. 11.

\section{CONClusion}

The integral feedback design has been found quite efficient to stabilize the laser beam on a very small detector area. The use of the dynamic output feedback with robust optimization techniques has permitted us to handel system and input uncertainties. Moreover, the developed approach seems be quite promising and efficient when the optical system may have other different configurations needed for laser communication.

\section{ACKNOWLEDGMENT}

This work is supported by the Deanship of Scientific Research (DSR) at KFUPM under grant, referenced KAUST004. The authors would like to express their thanks to the DSR for its support and assistance. 


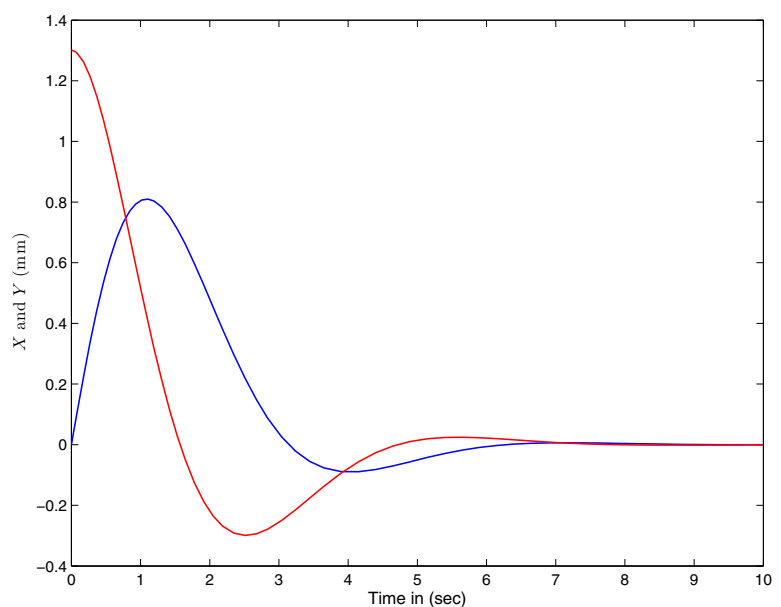

Fig. 9. The history of $X$ and $Y$

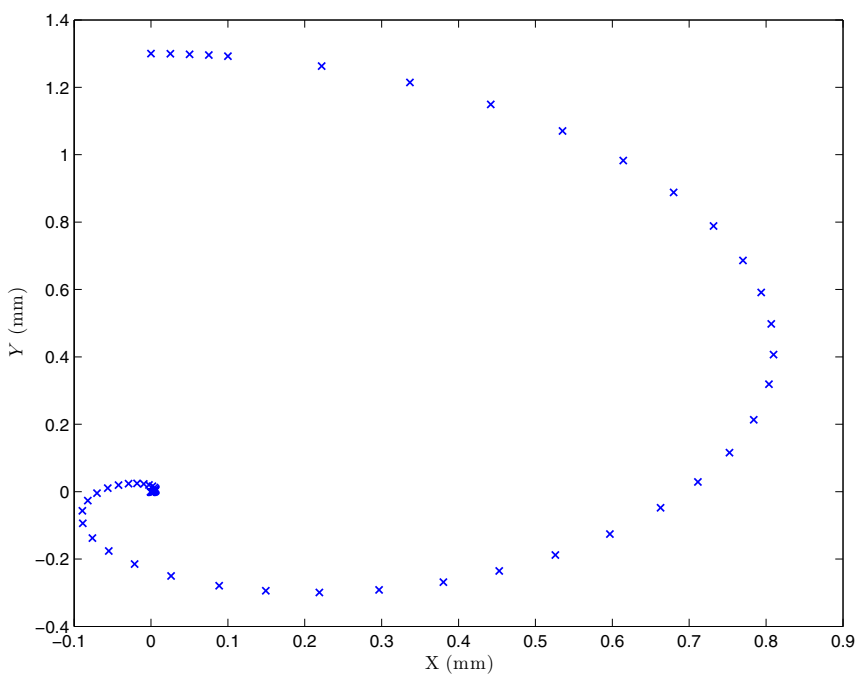

Fig. 10. The position of the beam spot on PSD

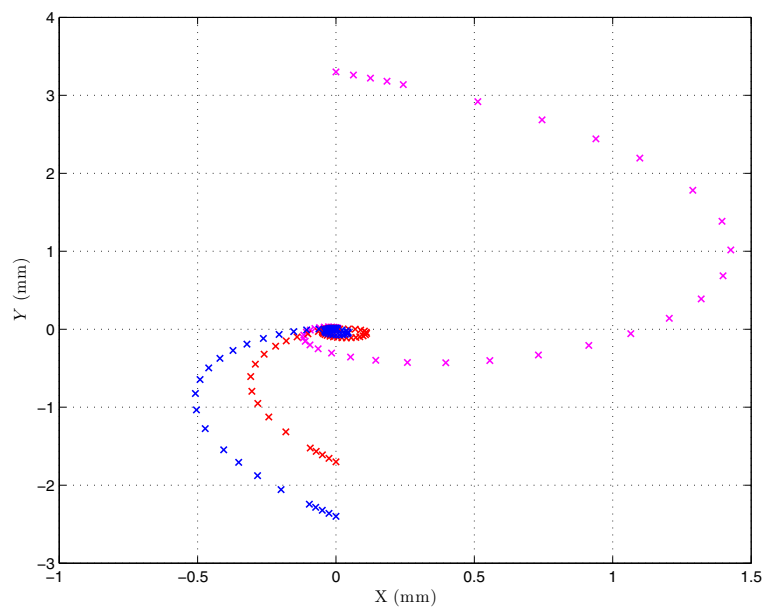

Fig. 11. The position of the beam spot for different initial conditions

\section{REFERENCES}

[1] J. A. Mynderse and G. T. C. Chiu, "Modeling of a dynamic mirror with antagonistic piezoelectric stack actuation," Journal of Dynamic Systems, Measurement, and Control, vol. 136, no. 2, pp. $041001-1$, 2013. [Online]. Available: http://dx.doi.org/10.1115/1.4025671

[2] G. Wang and F. Bai, "Robust tracking control of piezoelectric fast steering mirror with hysteresis and disturbances correction," in 34th Chinese Control Conference (CCC), July 2015, pp. 389-394.

[3] C. Wei, C. Sihai, W. Xin, and L. Dong, "A new two-dimensional fast steering mirror based on piezoelectric actuators," in 4th IEEE International Conference on Information Science and Technology, April 2014, pp. 308-311.

[4] W. Zhu, L. Bian, Y. An, G. Chen, and X. Rui, "Modeling and control of a two-axis fast steering mirror with piezoelectric stack actuators for laser beam tracking," Smart Materials and Structures, vol. 24, no. 7, p. 075014, 2015. [Online]. Available: http://stacks.iop.org/0964-1726/24/i=7/a=075014

[5] Y. Shao, D. L. Dickensheets, and P. Himmer, "3-D MOEMS mirror for laser beam pointing and focus control," IEEE Journal of Selected Topics in Quantum Electronics, vol. 10, no. 3, pp. 528-535, May 2004.

[6] M. Shao, Z. Wei, M. Hu, and G. Zhang, "Calibration method for a vision guiding-based laser-tracking measurement system," Measurement Science and Technology, vol. 26, no. 8, p. 085009, 2015. [Online]. Available: http://stacks.iop.org/0957-0233/26/i=8/a=085009

[7] H. Yoon, B. E. Bateman, and B. N. Agrawal, "Laser beam jitter control using recursive-least-squares adaptive filters," Journal of Dynamic Systems, Measurement, and Control, vol. 133, no. 8, pp. 041 001-1.

[8] T. Tsujimura, S. Muta, Y. Masaki, and K. Izumi, "Initial alignment scheme and tracking control technique of free space optics laser beam," in 5th International Conference on Optical Communication Systems (OPTICS), Aug 2014, pp. 1-6.

[9] S. Muta, T. Tsujimura, and K. Izumi, "Laser beam tracking system for active free-space optical communication," in Proceedings of the IEEE/SICE International Symposium on System Integration, Dec 2013, pp. 879-884.

[10] N. O. Perez-Arancibia, J. S. Gibson, and T. C. Tsao, "Observer-based intensity-feedback control for laser beam pointing and tracking," IEEE Transactions on Control Systems Technology, vol. 20, no. 1, pp. 31-47, Jan 2012.

[11] J. Y. Yen, C. S. Jeng, and K. C. Fan, "Servo design for a 3-D laser tracking measurement system," Transactions of the ASME Journal of Dynamic Systems, Measurement, and Control, vol. 118, no. 3, pp. 476481, Septemer 1996.

[12] A. Raj, J. A. V. Selvi, and S. Raghavan, "Terrestrial free space line of sight optical communication (TFSLSOC) using adaptive control steering system with laser beam tracking, aligning and positioning (ATP)," in International Conference on Wireless Communication and Sensor Computing (ICWCSC), Jan 2010, pp. 1-5.

[13] J. Zhou, C. Zhang, and C. Wen, "Robust adaptive output control of uncertain nonlinear plants with unknown backlash nonlinearity," IEEE Transactins on Automatic Control, vol. 52, no. 3, pp. 503-509, 2007.

[14] G. Tao and P. V. Kokotović, Adaptive control of systems with actuator and sensor nonlinearities, ser. Wiley series on adaptive and learning systems for signal processing, communications, and control. NewYork: John Wiley \& Sons, Inc., 1996. 